\title{
Compliance to radiation therapy of head and neck cancer patients and impact on treatment outcome
}

\author{
B. C. Ferreira ${ }^{1,2,6} \cdot$ P. Sá-Couto ${ }^{3}$ M. C. Lopes $^{4} \cdot$ L. Khouri $^{5}$
}

Received: 22 June 2015/Accepted: 25 September 2015/Published online: 12 October 2015

(C) Federación de Sociedades Españolas de Oncología (FESEO) 2015

\begin{abstract}
Aims The aims of the study were to evaluate head and neck cancer (HNC) patient's compliance to the planned radiation therapy (RT) using the department policy established in 2005 at IPOCFG and to estimate the impact on treatment outcome due to failure in receiving $\mathrm{RT}$ as prescribed.

Materials and methods $359 \mathrm{HNC}$ patients irradiated from 2007 to 2013 were included in this study. Patient cohort was divided into Group 1: patients receiving RT as prescribed and Group 2: patients that interrupted or suspended RT. Group Tox is the subgroup of patients that interrupted RT due to toxicity or intercurrent disease. Number and causes for treatment interruptions were assessed. The cumulative incidence of locoregional control (LRC), disease-free survival (DFS) and overall survival for Groups 1
\end{abstract}

Electronic supplementary material The online version of this article (doi:10.1007/s12094-015-1417-5) contains supplementary material, which is available to authorized users.

\section{B. C. Ferreira \\ bcf@estsp.ipp.pt}

1 I3N Physics Department, Aveiro University, Aveiro, Portugal

2 School of Allied Health Technologies, Polytechnic Institute of Porto, Porto, Portugal

3 Department of Mathematics, Center for Research and Development in Mathematics and Applications, Aveiro University, Aveiro, Portugal

4 Medical Physics Department, Portuguese Institute of Oncology of Coimbra Francisco Gentil, Coimbra, Portugal

5 Radiation Therapy Department, Portuguese Institute of Oncology of Coimbra Francisco Gentil, Coimbra, Portugal

6 Rua Valente Perfeito, 322, gab 20, 4400-330 Vila Nova de Gaia, Portugal and 2 was determined. Cox regression was performed to investigate potential hazard factors and logistic regression was made to determine risk factors related to treatment interruptions.

Results Major causes for treatment interruptions were toxicity plus intercurrent disease $(41.7 \%)$ and public holidays $(30.1 \%) . \quad 10.3 \%$ of the patients interrupted 3-9 days. Significant differences in survival distributions of the LRC between Groups 1 and 2, of up to $19 \%$, were found in the subgroup of patients with N2-3 tumours, for post-operative RT and for concomitant RT. Treatment breaks larger than two days had an almost fourfold increased risk of poorer LRC and DFS.

Conclusions Twin accelerators and treating on public holidays are effective measures minimizing RT breaks. For HNC, patient compliance is mostly limited by RT sideeffects. Efforts to maintain RT biological effective dose in HNC must be always undertaken.

Keywords Head and neck cancer - Radiation therapy · Treatment interruptions - Clinical outcome IMRT

\section{Introduction}

The influence of overall treatment time on outcome in RT of head and neck (HN) cancer is fairly well documented [1-3]. Treatment interruptions of 1 week resulted in losses in locoregional control of about $10 \%$ [1, 4]. Strategies to keep treatment effectiveness in the face of planned or unplanned interruptions have, therefore, been suggested [3, 5]. Transferring patients to a similar linac to overcome machine breaks or the delivery of two fractions per day, separated by at least a $6-8 \mathrm{~h}$ interval for sublethal damage to repair, are approaches to accomplish the prescribed 
overall treatment time. Alternatively, increasing the dose per fraction or the number of fractions are the strategies that have to be balanced against the risks of increased toxicity. To maintain biological treatment effectiveness in HN tumours, an extra dose of about 0.6-0.75 Gy per day of protraction would be required to counteract the effects of accelerated tumour repopulation [6].

At the Portuguese Institute of Oncology of Coimbra Francisco Gentil (IPOCFG), the causes for treatment interruptions for all patients treated in one treatment unit were assessed in 2005 [7]. Public holidays were the major cause for interruptions and most patients interrupted less than 5 days. Such study led to a change in department policy. First, patients were categorized by radiation oncologists. For those patients in whom breaks were likely to affect the outcome, treatments were delivered every day of the week despite holidays (except for Christmas and New Year's Day). Secondly, twin accelerators were acquired allowing the transfer of patients in case of malfunction or maintenance. For planned interruptions, if possible, overall treatment time is kept by treating on the weekend or by delivering two fractions per day from the same plan or from the boost plan. The cause for treatment interruption is then documented in the patient chart.

The first aim of this study was to assess compliance to the planned RT for $\mathrm{HN}$ cancer patients using the clinical protocol adopted since 2005 at IPOCFG [7]. Secondly, the aim of the study was to evaluate treatment outcome for this pathology, irradiated mostly with Intensity Modulated Radiation Therapy (IMRT), for patients that received RT as planned and, finally, to estimate the impact on treatment outcome from the lack of compliance to the prescribed treatment.

\section{Materials and methods}

\section{Patients and treatments}

From May 2007 to July 2013, 359 patients with HN squamous cell carcinoma $(90.0 \%)$ or undifferentiated carcinoma were treated at IPOCFG mostly with IMRT $(86 \%)$. Palliative and re-irradiated cases were not included in this cohort. All clinical patient data were collected by the radiation oncologists of the institute during the RT medical appointments. Thus, in this retrospective study, informed consent was not required. In this study, patient cohort was divided into two groups: Group 1 patients that received RT as prescribed (patients treated with multiple fractions per day or concomitant boost due to public holidays were included in this group, 12 cases); and Group 2 patients that failed to receive RT as planned, and were thus irradiated with a biological effective dose lower than prescribed, due to RT treatment breaks or incomplete treatment (69 and 11 cases, respectively). Ten patients that interrupted RT but received dose compensation were excluded from the survival analysis but were accounted for in the assessment of the causes for treatment interruptions. Mean follow-up time for Group 1 was 19.3 months [95\% confidence intervals (CI) 17.7-20.9 months] and for Group 2 was 22.5 months (95 \% CI 19.2-25.6 months). A subgroup of patients in the total cohort that interrupted RT due to toxicity or intercurrent disease (i.e. unrelated comorbidity) was created to evaluate the impact of these factors on treatment interruptions (Group Tox). This was composed of a total of 42 patients that interrupted treatment due to severe mucositis, radiodermitis, haematological toxicity, infection (e.g. pneumonia), anxiety, nausea/vomiting and mask intolerance. Patients and disease characteristics for all patients groups are summarized in Table 1.

Details on RT are described in detail elsewhere [8]. In summary, target volume delineation followed Gregoire et al.'s guidelines $[9,10]$. Prescription doses to primary tumour volume (post-operative or definitive) and large adenopathies ranged from 59.4 to $70.2 \mathrm{~Gy}$ and to high- and low-risk lymph nodes ranged from 50.4 to $59.4 \mathrm{~Gy}$. Concurrent chemotherapy was mainly cisplatin based. Patients unable to undergo this scheme were evaluated to cetuximab. Planning was performed in the Oncentra Treatment Planning System (Elekta/Nucletron). Simpler target volumes were irradiated with 3D conformal treatment techniques. More complex cases were irradiated with IMRT. Forwardly, optimized IMRT using a fractionation schedule of five fractions of $1.8 \mathrm{~Gy}$ per week was used from 2006 and progressively replaced since 2008 by inversely optimized IMRT (step-and-shoot Oncor Avant-Garde from Siemens) where dose integration of at least two prescription dose levels was used provided that a dose per fraction larger than 2.2 Gy was not obtained [11]. Dose assessment was based on collapsed-cone dose-computation algorithm for all patients.

\section{Statistical analysis}

Number and causes for treatment interruptions for this patient cohort were assessed. Multivariate logistic binary regressions were used to investigate factors that may be associated with interruption of RT due to toxicity or intercurrent disease. Adjusted odds ratios (adj. OR) and $95 \%$ CI were calculated for the factors: age, tumour site (hypopharynx + pharyngeal-laryngeal + larynx vs oral cavity vs oropharynx vs nasopharynx), stage $\mathrm{T}(1-2$ vs $2-3)$ and $\mathrm{N}(0-1$ vs $2-3)$, type of RT (concomitant vs non-concomitant) and type of treatment (post-operative vs definitive). 
Table 1 Patient and treatment characteristics

\begin{tabular}{|c|c|c|c|c|}
\hline Characteristics & Total, $N(\%)$ & Group $1, N(\%)$ & Group 2, $N(\%)$ & Group Tox, $N(\%)$ \\
\hline \multicolumn{5}{|l|}{ Age } \\
\hline$\leq 55$ & $150(42.0)$ & $104(38.7)$ & $41(51.3)$ & $20(47.6)$ \\
\hline$>55$ & $209(58.2)$ & $165(61.3)$ & $39(48.8)$ & $22(52.4)$ \\
\hline \multicolumn{5}{|l|}{ Gender } \\
\hline Male & $304(84.7)$ & $236(87.7)$ & $61(76.3)$ & $29(69.0)$ \\
\hline Female & $55(15.3)$ & $33(12.3)$ & $19(23.8)$ & $13(31.0)$ \\
\hline \multicolumn{5}{|l|}{ Site } \\
\hline Larynx & $85(23.7)$ & 67 (24.9) & $18(22.5)$ & $7(16.7)$ \\
\hline Oral cavity & $71(19.8)$ & $58(21.6)$ & $12(15.0)$ & $8(19.0)$ \\
\hline Oropharynx & $70(19.5)$ & $49(18.2)$ & $17(21.3)$ & $12(28.6)$ \\
\hline Nasopharynx & $45(12.5)$ & $31(11.5)$ & $11(13.8)$ & $10(23.8)$ \\
\hline Pharyngeal-laryngeal & $39(10.9)$ & $28(10.4)$ & $10(12.5)$ & $1(2.4)$ \\
\hline Hypopharynx & $27(7.5)$ & $18(6.7)$ & $8(10.0)$ & $3(7.1)$ \\
\hline Others & $22(6.1)$ & $18(6.7)$ & $4(5.0)$ & $1(2.4)$ \\
\hline \multicolumn{5}{|l|}{$\mathrm{T}$ stage } \\
\hline $1-2$ & $187(52.1)$ & $151(56.1)$ & $32(40.0)$ & $15(35.7)$ \\
\hline $3-4$ & $172(47.9)$ & $118(43.9)$ & $48(60.0)$ & $27(64.3)$ \\
\hline \multicolumn{5}{|l|}{ N stage } \\
\hline $0-1$ & $158(44.0)$ & $133(49.4)$ & $23(28.8)$ & $10(23.8)$ \\
\hline $2-3$ & $201(56.0)$ & $136(50.6)$ & $57(71.3)$ & $32(76.2)$ \\
\hline \multicolumn{5}{|l|}{ Type treatment } \\
\hline Post-operative & $145(40.4)$ & $119(44.2)$ & $24(30.0)$ & $10(23.8)$ \\
\hline Definitive & $214(59.6)$ & $150(55.7)$ & $56(70.0)$ & $32(76.2)$ \\
\hline \multicolumn{5}{|l|}{ Type of RT } \\
\hline Non-concomitant & $191(53.2)$ & $157(58.4)$ & $31(38.7)$ & $12(28.6)$ \\
\hline Concomitant & $168(46.8)$ & 112 (41.6) & $49(61.3)$ & $30(71.4)$ \\
\hline \multicolumn{5}{|l|}{ RT technique } \\
\hline 3DCRT & $52(14.5)$ & $46(17.1)$ & $6(7.5)$ & $1(2.4)$ \\
\hline fIMRT & $98(27.3)$ & 77 (28.6) & $20(25.0)$ & $6(14.3)$ \\
\hline IMRT & 209 (58.2) & $146(54.3)$ & $54(67.5)$ & $35(83.4)$ \\
\hline
\end{tabular}

Group 1 is the group of patients that received RT in the planned overall treatment time, Group 2 are the patients with extended treatment time or suspended RT and Group Tox is the group of patients, from the total cohort, that interrupted RT due to toxicity or intercurrent disease. 10 patients that received extra fractions to compensate for RT interruptions were not included in Group 1 or 2, but were accounted for in the total

3DCRT 3D Conformal RT, fIMRT forward optimized IMRT and IMRT refers to inversely optimized Intensity Modulated RT

Locoregional control was defined from the time complete tumour response to the initial treatment protocol was obtained up to the time of recurrence. Complete tumour response was achieved in $80.2 \%$ of the patients. Diseasefree survival was defined from the time since complete tumour response to the therapy has been achieved up to the time of recurrence, metastasis, second tumour or death, independently of appearance order. The rate of distant metastasis and the rate of overall survival were calculated from the start of RT. The cumulative incidence of locoregional control, disease-free survival, distant metastasis and overall survival were calculated using the Kaplan-Meier method for the total population cohort and stratifying the variables (one at a time): age, gender, $\mathrm{T}$ stage and $\mathrm{N}$ stage, type of treatment (post-operative vs definitive) and type of RT (concomitant vs non-concomitant). Log-rank test was used to test the equality of the survival distributions between Groups 1 and 2.

Potential prognostic factors [hazard ratios (HR) and the correspondent $95 \% \mathrm{CI}$ ] with association with locoregional control, disease-free survival and overall survival were explored in univariate and multivariate analysis performed using Cox regression models. These were: age (continuous variable), gender, tumour site (hypopharynx + pharyngeal-laryngeal + larynx vs oropharynx vs oral cavity), $\mathrm{T}$ stage (1-2 vs $3-4)$ and $\mathrm{N}$ stage (0-1 vs $2-3$ ), type of RT 
Table 2 Percentage of treatment breaks by duration of gap and results of the multivariate logistic regression for treatment interruptions due to RT toxicity or intercurrent disease (interc dis)

\begin{tabular}{|c|c|c|c|c|c|c|c|c|}
\hline \multirow[t]{2}{*}{ Duration of gap/days } & \multicolumn{5}{|l|}{$N / \%$} & \multicolumn{3}{|c|}{ Toxicity + interc dis } \\
\hline & 0 & $1-2$ & $3-5$ & $5-9$ & Inc. RT & Adj. OR & $95 \% \mathrm{CI}$ & $p$ \\
\hline Total & 74.9 & 11.7 & 7.5 & 2.8 & 3.1 & & & \\
\hline \multicolumn{9}{|l|}{ Tumour site } \\
\hline Hypop + PhL + Larynx & 74.8 & 9.3 & 9.3 & 2.0 & 3.3 & 1 & - & - \\
\hline Oral cavity & 81.7 & 7.0 & 4.2 & 2.8 & 4.2 & 2.09 & {$[0.74 ; 5.91]$} & 0.165 \\
\hline Oropharynx & 70.0 & 17.1 & 4.3 & 4.3 & 4.3 & 2.76 & {$[1.07 ; 7.10]$} & $\mathbf{0 . 0 3 5}$ \\
\hline Nasopharynx & 62.2 & 17.8 & 15.6 & 4.4 & 0.0 & 2.59 & {$[0.91 ; 7.36]$} & 0.075 \\
\hline \multicolumn{9}{|l|}{ T stage } \\
\hline $1-2$ & 80.2 & 10.2 & 5.9 & 1.1 & 2.7 & 1 & - & - \\
\hline $3-4$ & 69.2 & 13.4 & 9.3 & 4.7 & 3.5 & 2.86 & {$[1.29 ; 6.33]$} & 0.009 \\
\hline \multicolumn{9}{|l|}{$\mathrm{N}$ stage } \\
\hline $0-1$ & 84.2 & 8.2 & 5.1 & 1.9 & 0.6 & 1 & - & - \\
\hline $2-3$ & 67.7 & 14.4 & 9.5 & 3.5 & 5.0 & 2.07 & {$[0.87 ; 4.96]$} & 0.102 \\
\hline \multicolumn{9}{|l|}{ Type RT } \\
\hline No-concomitant & 82.2 & 9.4 & 5.8 & 0.5 & 2.1 & 1 & - & - \\
\hline Concomitant & 66.7 & 14.3 & 9.5 & 5.4 & 4.2 & 1.96 & {$[0.83 ; 4.60]$} & 0.124 \\
\hline \multicolumn{9}{|l|}{ Type treatment } \\
\hline Post-operative & 82.8 & 5.5 & 4.8 & 2.8 & 4.1 & 1 & - & - \\
\hline Definitive & 69.6 & 15.9 & 9.3 & 2.8 & 2.3 & 1.42 & {$[0.55 ; 3.67]$} & 0.473 \\
\hline
\end{tabular}

Results for: age (toxicity + intercurrent disease) adj. OR $=1.00,95 \%$ CI $[0.97 ; 1.03], p=0.811$

Inc. RT shows the percentage of patients that suspended RT. Concomitant refers to patients that received cisplatin or cetuximab concomitantly with RT while non-concomitant refers to other cases

Bold values are statistically significant

Adj. OR adjusted odd ratio and $95 \% \mathrm{CI}$ for its $95 \%$ confidence intervals, Hypop + PhL hypoharynx and pharyngeal-laryngeal tumours (concomitant vs non-concomitant), type of treatment (postoperative vs definitive), prescribed dose ( $\sim 59$ vs $\sim 65$ vs $\sim 70 / \mathrm{Gy}$ ), dose per fraction ( $\leq 2$ vs $>2 \mathrm{~Gy}$ ), overall treatment time (continuous variable) and duration of the gap (0 vs 1-2 days vs $>2$ days). Due to the high correlation between input variables, overall treatment time and prescribed dose were excluded from the analysis.

All statistical analyses were performed using SPSS $^{\circledR}$ Software, version 20.0 (SPSS, Inc., Chicago, IL, USA) and $p$ values under 0.05 were considered significant.

\section{Results}

The major cause for unplanned treatment interruptions was RT toxicity plus intercurrent disease (41.7\%). Replanning, due to patient weight loss, was the cause for the treatment interruption in six patients $(5.8 \%)$ by a maximum of 2 days. Public holiday (Christmas and New Year) was the major cause for planned treatment interruptions $(30.1 \%)$. Machine maintenance or breakdown was the cause for the interruption in only five patients (4.9\%) (Supplementary material, Table 1).

Median overall treatment time for Group 1 was $44.0 \pm 3.7$ days (range 37-54 days) and for Group 2 was
$47.0 \pm 4.9$ days (range 35-61 days). Median overall treatment time for patients with extended delivery time was $47.0 \pm 4.4$ days (range 39-61 days) and for patients with incomplete treatment was $42.0 \pm 4.6$ days (range 35-49 days).

In Table 2, the percentage of treatment interruptions by the duration of the gap is shown for all patients. Almost $75 \%$ of all patients received RT in the planned schedule or in less time ( 9 cases). The shorter overall treatment time was due to the delivery of multiple fractions per day or concomitant boost to compensate for planned treatment interruptions. The maximum number of days of interruption was 9 days. From the patients that interrupted, $11.7 \%$ interrupted 1 or 2 days, $7.5 \%$ interrupted 3-5 days and $2.8 \%$ (10 cases) interrupted more than 5 days. $3.1 \%$ of the patients did not complete RT due to severe RT toxicity and intercurrent disease.

More than $80 \%$ of the patients with earlier stages of the disease received their treatment as planned compared to around $68 \%$ of the patients with more advanced tumour stages (Table 2). The percentage of patients that interrupted more than 3 days with T1-2 and T3-4 tumours was 7.0 and $14 \%$, respectively. Risk factors significantly associated with treatment interruption due to RT toxicity or 

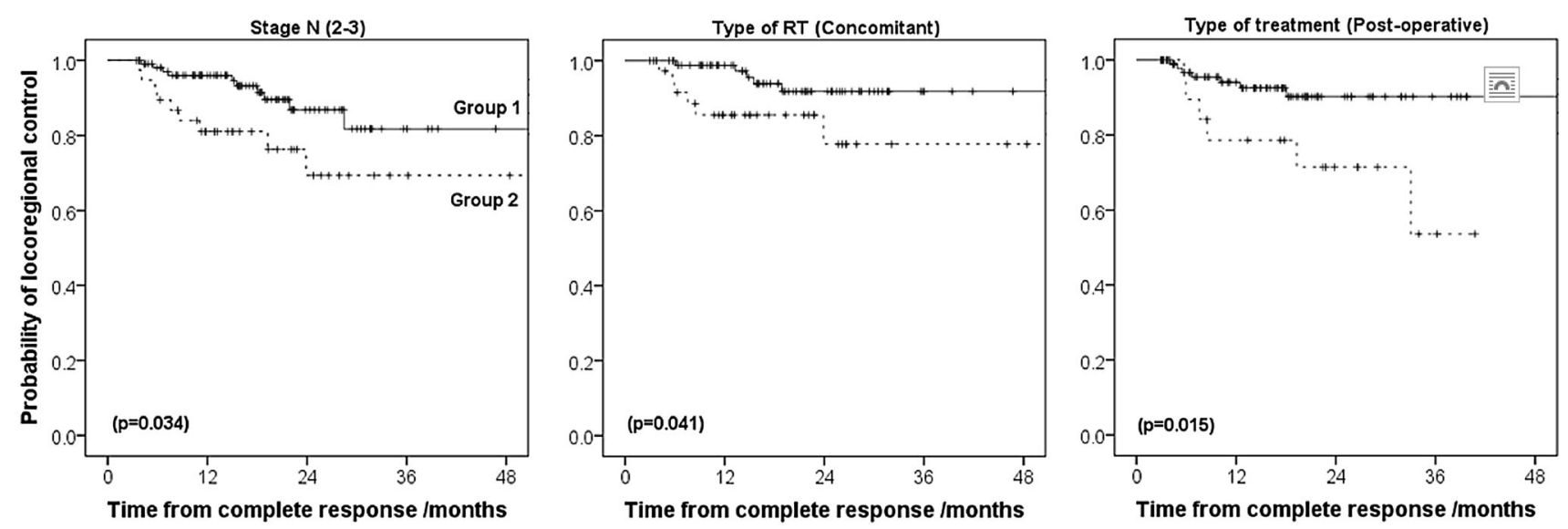

Fig. 1 Cumulative incidence of: locoregional control for patients that complied with idealized overall treatment time (Group 1) and patients with extended treatment time or incomplete treatment (Group 2) for the subgroups: stage N2-3, concomitant RT and post-operative RT, respectively

intercurrent disease were $\mathrm{T}$ stage and oropharynx tumours (Table 2).

At 24 months, locoregional control for Group 1 and for Group 2 was 87.0 and $76.7 \%$, disease-free survival was 71.2 and $60.0 \%$, distant metastases rate was 24.6 and $27.4 \%$ and overall survival was 73.0 and $62.9 \%$, respectively. Although Group 1 when compared with Group 2 always presented better results in all studied endpoints, differences in the probability distribution were not statistically significant.

Significant differences in the Kaplan-Meier survival curves for the probability of locoregional control between Groups 1 and 2 were obtained for the subgroups: stage N3-4, concomitant RT and for post-operative RT (Fig. 1). At 24 months, locoregional for Group 1 vs Group 2 for patients with N2-3 tumours was 86.9 vs $69.4 \%$, for concomitant RT was 91.8 vs $77.7 \%$ and for post-operative RT was 90.2 vs $71.5 \%$, respectively. Differences in the probability of locoregional control between Groups 1 and 2 were also obtained for the subgroups stage T1-2 and T2-3 but without reaching statistical significance. Similarly, no statistical significant differences were obtained for the endpoints disease-free survival or overall survival for all evaluated subgroups (Supplementary material-Table 2).

In Table 3, the variables significantly associated with the probability of locoregional control, disease-free survival and overall survival in univariate and multivariate analysis are shown. Duration of the gap superior to 2 days was significantly related to an increased risk of tumour recurrence and poorer disease-free survival both in univariate analysis and multivariate analysis (HR for LRC and DFS 3). T stage was significantly associated with an increased risk of death both in univariate and multivariate analysis (HR 1.84) while $\mathrm{N}$ stage was significantly related to worse disease-free survival and overall survival ( HR 2). Inoperable tumour cases undergoing definitive RT, compared to post-operative RT, were significantly related with worst locoregional control (HR 3.25) and disease-free survival (HR 2.49).

\section{Discussion}

The fast repopulation rate of HN tumours cells occurring during RT makes overall treatment time one of the most important factors affecting the probability of tumour control $[1-3,6,12]$. To improve patient compliance to the prescribed treatment time, new strategies were adopted at IPOCFG [7]. With these policies, almost $75 \%$ of the HN cancer patients in this cohort received their treatment as planned (Table 2). Excluding the patients who interrupted RT due to complications, therapy for these patients was delivered as prescribed to almost $88 \%$ of the patients.

The most important factors that minimized the rate of the department-related RT interruptions were: first, twin linear accelerators allowed the safe delivery of the planned treatments and second, the delivery of RT 5 days a week in priority patients (with the exception of Christmas and New Year's days). The number of interruptions due to machine maintenance and breakdown was reduced from $43 \%$ before 2005 to $4.9 \%$. Some minor organization issues are always needed to reschedule non-priority patients. However, transfer of priority patients between dosimetrically identical machines completely eliminated all workload related with replanning for a different machine. With the conversion of almost all public holidays into working days, a reduction of interruptions due to holidays from 49 to $30 \%$ was possible. In half of the cases, interruptions were compensated by the delivery of multiple fractions per day, concomitant boost, treatments on the weekend or an increase in the number of fractions delivered. Even so, overall treatment time for patients that interrupted their treatment due to public holidays was in average 3 days 
Table 3 Results from Cox regression analysis for the endpoints locoregional control, disease-free survival and overall survival

\begin{tabular}{|c|c|c|c|c|c|c|}
\hline & \multicolumn{3}{|c|}{ Univariate analysis } & \multicolumn{3}{|c|}{ Multivariate analysis } \\
\hline & HR & $95 \% \mathrm{CI}$ & $p$ & HR & $95 \% \mathrm{CI}$ & $p$ \\
\hline \multicolumn{7}{|c|}{ Locoregional control } \\
\hline \multicolumn{7}{|l|}{ Type treatment } \\
\hline Post-operative & 1 & - & - & 1 & - & - \\
\hline Definitive & 1.75 & {$[0.79 ; 3.88]$} & 0.172 & 3.25 & {$[1.12 ; 9.48]$} & $\mathbf{0 . 0 3 0}$ \\
\hline \multicolumn{7}{|l|}{ Duration of gap } \\
\hline 0 & 1 & - & - & 1 & - & - \\
\hline $1-2$ days & 2.16 & {$[0.80 ; 5.88]$} & 0.130 & 1.82 & {$[0.65 ; 5.09]$} & 0.251 \\
\hline$>2$ days & 2.99 & {$[1.01 ; 8.92]$} & 0.049 & 3.98 & {$[1.12 ; 14.15]$} & $\mathbf{0 . 0 3 3}$ \\
\hline \multicolumn{7}{|c|}{ Disease-free survival } \\
\hline \multicolumn{7}{|l|}{$\mathrm{N}$ stage } \\
\hline $0-1$ & 1 & - & - & 1 & - & - \\
\hline $2-3$ & 1.95 & {$[1.14 ; 3.32]$} & 0.014 & 2.03 & {$[1.09 ; 3.80]$} & 0.026 \\
\hline \multicolumn{7}{|l|}{ Type treatment } \\
\hline Post-operative & 1 & - & - & 1 & - & - \\
\hline Definitive & 1.92 & {$[1.13 ; 3.27]$} & 0.016 & 2.49 & {$[1.24 ; 5.00]$} & 0.010 \\
\hline \multicolumn{7}{|l|}{ Duration of gap } \\
\hline 0 & 1 & - & - & 1 & - & - \\
\hline 1-2 days & 1.33 & {$[0.62 ; 2.82]$} & 0.464 & 1.17 & {$[0.54 ; 2.53]$} & 0.695 \\
\hline$>2$ days & 2.53 & {$[1.19 ; 5.38]$} & 0.016 & 3.05 & {$[1.31 ; 6.97]$} & 0.009 \\
\hline \multicolumn{7}{|l|}{ Overall survival } \\
\hline \multicolumn{7}{|l|}{$\mathrm{T}$ stage } \\
\hline $1-2$ & 1 & - & - & 1 & - & - \\
\hline $3-4$ & 2.33 & {$[1.33 ; 4.08]$} & 0.003 & 1.84 & {$[1.02 ; 3.34]$} & 0.042 \\
\hline \multicolumn{7}{|l|}{$\mathrm{N}$ stage } \\
\hline $0-1$ & 1 & - & - & 1 & - & - \\
\hline $2-3$ & 2.62 & {$[1.45 ; 4.76]$} & 0.002 & 2.20 & {$[1.15 ; 4.21]$} & 0.018 \\
\hline \multicolumn{7}{|l|}{ Type treatment } \\
\hline Post-operative & 1 & - & - & 1 & - & - \\
\hline Definitive & 1.56 & {$[0.93 ; 2.62]$} & 0.090 & 1.43 & {$[0.71 ; 2.88]$} & 0.312 \\
\hline \multicolumn{7}{|l|}{ Duration of gap } \\
\hline 0 & 1 & - & - & 1 & - & - \\
\hline 1-2 days & 1.42 & {$[0.69 ; 2.90]$} & 0.342 & 1.11 & {$[0.53 ; 2.33]$} & 0.791 \\
\hline$>2$ days & 1.86 & {$[0.91 ; 3.80]$} & 0.092 & 1.53 & {$[0.72 ; 3.25]$} & 0.272 \\
\hline
\end{tabular}

Other variables considered $(p>0.10)$ : age, gender, tumour site, dose per fraction, type of RT Bold values are statistically significant

$H R$ hazard ratio and $95 \% \mathrm{CI}$ for its $95 \%$ confidence intervals longer than prescribed. Further measures to avoid loss in treatment outcome as a result of the extension in overall treatment time due to Christmas holidays may thus still be needed.

Domestic reasons and replanning had a smaller impact on the number of treatment interruptions than departmentrelated causes. In most cases, personal motives for missing the treatment were not documented. Strike on public transports, holidays and private celebrations were some of the reasons reported by patients for non-compliance. With the availability of tools for adaptive RT, an increasing number of cases may benefit from replanning. In this cohort, in $86 \%$ of the cases in which adaptive RT was applied, treatment was resumed without interruption. In case treatment interruption was unavoidable, the duration of the gap did not exceed 2 days. If the therapeutic advantages of adaptive RT [13] outweigh the losses from an extended overall treatment time still needs to be determined. Better patient and staff education and closer patient follow-up may contribute to reduce, or ideally eliminate, the number of treatment breaks due to domestic reasons and adaptive RT.

Patients with inoperable advanced $\mathrm{HN}$ tumours are generally advised for concomitant definitive RT. Larger RT 
doses imply a longer overall treatment time raising the odds for treatment interruptions due to department-related causes. Patients with more advanced tumour stages undergoing definitive RT were less likely to receive treatment in the idealized time (Table 2). Also, the duration of the treatment break was longer for such patients. More aggressive treatments delivered with definitive concomitant RT, mostly, with cisplatin are generally associated with increased patient toxicity. Compliance to the planned RT may then become more difficult. However, despite the increased risk for treatment interruptions due to toxicity or intercurrent disease for advanced HN tumour cases treated with definitive RT and concomitant RT, these were not statistically associated with interruption of RT due to comorbidity (Table 2-right column). Only advanced T stage and oropharynx tumour cases remained significantly related to RT interruption due to RT toxicity plus intercurrent disease.

The new department policies significantly raised the number of patients receiving RT as planned compared to the compliance obtained before 2005. Even so, comparing survival estimates for patients receiving RT as prescribed against patients that failed in receiving the planned treatment, differences up to $10 \%$ in locoregional control, disease-free survival and overall survival between the two groups were obtained. None of these differences were statistically significant and, therefore, a longer follow-up is needed to strengthen the findings of this study limited by the small number of events in each group and its retrospective nature.

By stratifying the population into subcategories, statistically significant differences in the probability of locoregional control between Groups 1 and 2 for patients with advanced $\mathrm{N}$ stage tumours, patients receiving post-operative RT and for the subgroup undergoing concomitant chemoradiation were obtained (Fig. 1). The difference in mean overall treatment time between Groups 1 and 2 for each subcategory ranged from 2 to 3 days, resulting in differences in the probability of locoregional control at 2 years of 14-19\%. Shaverdian et al. [14] reported that for cervical cancer extended overall treatment time had no impact on patients receiving concomitant chemoradiation. However, Cannon et al. [15] showed that this was not the case for head and neck tumours. In this study, the therapeutic benefits obtained with multimodality treatments, through surgery or concomitant chemotherapy combined with RT, were lost when RT was not delivered uninterruptedly (Supplementary material-Table 2). Efforts to deliver RT as planned to HN cancer patients should, therefore, be always undertaken to maximize treatment success.

Suwinski et al. [1] reported that a protraction in RT of post-operative head and neck tumour patients of 10 days resulted in a 10-20\% difference in recurrence-free survival. Interestingly, these differences were obtained for treatments longer than 45 days and for gap durations longer than 5 days. In this study, no patient interrupted more than 9 days and the number of patients with a gap larger than 2 days was reduced from about $46 \%$ [7] to $10.3 \%$ using the new department policies. For the survival endpoints investigated, the hazard risk of treatment failure increased with the duration of the RT break (Table 3). An interruption longer than just 2 days was significantly associated with poorer locoregional control and diseasefree survival increasing the risk of treatment failure by a factor of three to four. RT gaps larger than 2 days should, therefore, be strongly avoided. Actions to keep overall treatment time and the biological effective dose in $\mathrm{HN}$ tumour cases should always be adopted if treatment interruptions are unavoidable.

\section{Conclusion}

The purchase of twin accelerators and treating on public holidays has succeeded to significantly reduce departmentrelated RT interruptions. For RT of HN tumour cases, compliance to the planned treatment is mostly limited by the severity of acute side-effects. Preventing measures to minimize mucositis, dysphagia and pain during RT should, therefore, be adopted especially in patients treated with advanced tumours (especially oropharynx tumours cases). In the face of inevitable treatment interruptions, the duration of the gap should be minimized and kept under 2 days. A protraction in overall treatment time by more than 2 days increased the relative risk of treatment failure by three to four times compared to the delivery of RT without interruptions. Strategies to keep prescribed biological effectiveness in RT of HN tumours should, therefore, be always adopted.

Acknowledgments This work was financed by the Foundation for Science in Portugal with the program Pest-C/CTM/LA0025/2011. P. Sá-Couto's work was supported by Portuguese funds through the CIDMA-Center for Research and Development in Mathematics and Applications, and the Portuguese Foundation for Science within project Pest-OE/MAT/UI4106/2014.

\section{Compliance with ethical standards}

Conflict of interest The authors have no conflicts of interest.

\section{References}

1. Suwinski R, Sowa A, Rutkowski T, Wydmanski J, Tarnawski R, Maciejewski B. Time factor in postoperative radiotherapy: a multivariate locoregional control analysis in 868 patients. Int J Radiat Oncol Biol Phys. 2003;56(2):399-412.

2. Groome PA, O'Sullivan B, Mackillop WJ, Jackson LD, Schulze K, Irish JC et al. Compromised local control due to treatment interruptions and late treatment breaks in early glottic cancer: population-based outcomes study 
supporting need for intensified treatment schedules. Int J Radiat Oncol Biol Phys. 2006;64(4):1002-12.

3. Bese NS, Hendry J, Jeremic B. Effects of prolongation of overall treatment time due to unplanned interruptions during radiotherapy of different tumor sites and practical methods for compensation. Int $\mathbf{J}$ Radiat Oncol Biol Phys. 2007;68(3):654-61.

4. Fowler JF. Biological factors influencing optimum fractionation in radiation therapy. Acta Oncol. 2001;40(6):712-7.

5. Garau M, Monné J, Serés MJ. Monfà Binefa C, Peraire Llopis M. Compliance to the prescribed overall treatment time (OTT) of curative radiotherapy in normal clinical practice and impact on treatment duration of counteracting short interruptions by treating patients on Saturdays. Clin Transl Oncol. 2009;11(5):302-11.

6. Tarnawski R, Fowler J, Skladowski K, Swierniak A, Suwinski R, Maciejewski $\mathrm{B}$, et al. How fast is repopulation of tumor cells during the treatment gap? Int J Radiat Oncol Biol Phys. 2002;54(1):229-36.

7. Andrade L, Pais M. Lopes MC unsheduled interruptions and prolongation of treatment time in radiotherapy—one year study. Radiother Oncol. 2005;76(Supp. 2):S79.

8. Ferreira BC, Marques RV, Khouri L, Santos T, Sá-Couto P, Lopes MC Assessment and topographic characterization of locoregional recurrences in head and neck tumours. Radiat. Oncol. 2015;10:41.

9. Grégoire V, Levendag P, Ang KK, Bernier J, Braaksma M, Budach V, et al. CTbased delineation of lymph node levels and related CTVs in the node-negative neck: DAHANCA, EORTC, GORTEC, NCIC. RTOG consensus guidelines. Radiother Oncol. 2003;69(3):227-36.

10. Grégoire V, Eisbruch A, Hamoir M, Levendag P. Proposal for the delineation of the nodal CTV in the node-positive and the post-operative neck. Radiother Oncol. 2006;79(1):15-20.

11. Ferreira BC, Lopes MC, Mateus J, Capela M, Mavroidis P. Radiobiological evaluation of forward and inverse IMRT using different fractionations for head and neck tumours. Radiat. Oncol. 2010;5:57.

12. Fesinmeyer MD, Mehta V, Blough D, Tock L, Ramsey SD. Effect of radiotherapy interruptions on survival in medicare enrollees with local and regional head-and-neck cancer. Int J Radiat Oncol Biol Phys. 2010;78(3):675-81.

13. Zhao L, Wan Q, Zhou Y, Deng X, Xie C, Wu S. The role of replanning in fractionated intensity modulated radiotherapy for nasopharyngeal carcinoma. Radiother Oncol. 2011;98(1):23-7.

14. Shaverdian N, Gondi V, Sklenar KL, Dunn EF, Petereit DG, Straub MR, et al Effects of treatment duration during concomitant chemoradiation therapy for cervical cancer. Int J Radiat Oncol Biol Phys. 2013;86(3):562-8.

15. Cannon DM, Geye HM, Hartig GK, Traynor AM, Hoang T, McCulloch TM, et al. Increased local failure risk with prolonged radiation treatment time in head and neck cancer treated with concurrent chemotherapy. Head Neck. 2014;36(8):1120-5. 